\title{
Core-Shell Magnetic Morphology of Structurally Uniform Magnetite Nanoparticles
}

\author{
K. L. Krycka, ${ }^{1}$ R. A. Booth, ${ }^{2}$ C. R. Hogg, ${ }^{2}$ Y. Ijiri, ${ }^{3}$ J. A. Borchers, ${ }^{1}$ W. C. Chen, ${ }^{1,4}$ S. M. Watson, ${ }^{1}$ M. Laver, ${ }^{5}$ T. R. Gentile, ${ }^{1}$ \\ L. R. Dedon, ${ }^{3}$ S. Harris, ${ }^{3}$ J. J. Rhyne, ${ }^{6}$ and S. A. Majetich ${ }^{2}$ \\ ${ }^{1}$ NIST Center for Neutron Research, National Institute of Standards and Technology, Gaithersburg, Maryland 20899, USA \\ ${ }^{2}$ Department of Physics, Carnegie Mellon University, Pittsburgh, Pennsylvania 15213, USA \\ ${ }^{3}$ Department of Physics and Astronomy, Oberlin College, Oberlin, Ohio 44074, USA \\ ${ }^{4}$ Department of Materials Science and Engineering, University of Maryland, College Park, Maryland 20742-2115, USA \\ ${ }^{5}$ Paul Scherrer Institute, Villigen, Switzerland \\ ${ }^{6}$ Los Alamos National Laboratory, Los Alamos, New Mexico 87545, USA
}

(Received 25 February 2010; published 19 May 2010)

\begin{abstract}
A new development in small-angle neutron scattering with polarization analysis allows us to directly extract the average spatial distributions of magnetic moments and their correlations with threedimensional directional sensitivity in any magnetic field. Applied to a collection of spherical magnetite nanoparticles $9.0 \mathrm{~nm}$ in diameter, this enhanced method reveals uniformly canted, magnetically active shells in a nominally saturating field of $1.2 \mathrm{~T}$. The shell thickness depends on temperature, and it disappears altogether when the external field is removed, confirming that these canted nanoparticle shells are magnetic, rather than structural, in origin.
\end{abstract}

DOI: 10.1103/PhysRevLett.104.207203

PACS numbers: 75.70.- $-\mathrm{i}$

Monodomain magnetic nanoparticles are an important prototype for understanding the behavior of magnetic recording media, ferrofluids, and beads for magnetic separation of biological species. While it is tempting to assume that the moments are uniformly aligned within each nanoparticle, their spin configurations are typically far more complex [1]. When the particles contain both ferromagnetic and antiferromagnetic phases, exchange bias effects may occur [2-5]. Surface disorder can lead to a magnetically dead layer or a spin glasslike phase [6-9]. Surfaces can also introduce a cubic anisotropy term when the material otherwise has uniaxial anisotropy [10]. Surface anisotropy has led to predictions of a "hedgehog" configuration where the spins spike outward normal to the surface [11-14], an "artichoke" confirmation where they are parallel to the surface and directed from one pole to the other [13], and a "throttled" configuration where the spins have been tilted slightly inward at the south pole and outward at the north pole [13]. Experimental verification of these theoretical predictions is extremely challenging because the magnetization from the distorted surface spins typically averages out for macroscopic measurements. Here we extend the capability of small-angle neutron scattering (SANS) with polarization analysis in order to directly measure the ordered magnetic structure perpendicular to an applied field, thus allowing us to "see" for the first time the magnetically distinctive core-shell morphology. We demonstrate that magnetic shells 1.0 to $1.5 \mathrm{~nm}$ thick, oriented $90^{\circ}$ perpendicular to their ferrimagnetic cores, form between 160 and $320 \mathrm{~K}$ under conditions of nominal saturation at $1.2 \mathrm{~T}$, yet, vanish in the absence either of an applied field or interparticle interactions.

To minimize structural disorder, we have focused our SANS investigation with polarization analysis on $9.0 \mathrm{~nm}$ magnetite nanoparticles prepared by high temperature chemical methods [15]. These particles are monodisperse and can be self-assembled into face-centered cubic (FCC) superlattices or nanoparticle crystals [16]. The use of nanoparticle crystals leads to enhanced scattering at Bragg peaks, and the overall structural uniformity of the sample enables precise determination of the magnetic length scales.

Polarization analyzed SANS is ideal for discriminating among proposed models because it can detect the distribution and orientation of magnetic structures, even buried features, with subnanometer resolution. With the neutron polarization spin state as + or - , measurement of all four neutron spin cross-sections $(++,+-,-+$, and -- ) allows for the unique separation of nuclear scattering $\left(N^{2}\right)$ from magnetic scattering, irrespective of whether the sample is magnetically saturated. Application of polarization analysis to SANS [17-19] has been very limited since it requires a polarization analyzer capable of capturing a divergent, 2D, scattered beam, such as a polarized ${ }^{3} \mathrm{He}$ spin filter [20]. Additionally, we expand beyond the formalism of previous work [18] to directly measure the magnetic structure from moments oriented perpendicular to the applied field $\left(M_{Y}^{2}+M_{Z}^{2}=2 M_{\text {PERP }}^{2}\right)$, as well as the moments parallel to the applied magnetic field $\left(M_{X}^{2}=\right.$ $\left.M_{\text {PARL }}\right)$. This provides a true 3D measurement of magnetic structure. Building upon our initial investigation of magnetite nanoparticles in zero field [21] where $M_{\text {PARL }}^{2}$ and $M_{\text {PARP }}^{2}$ were equivalent, here we examine the magnetic response within a high applied field where $M_{\mathrm{PERP}}^{2}$ is distinctly different from $M_{\mathrm{PARL}}^{2}$.

Our setup is shown in Fig. 1(a) with the incoming beam along $Z$, the applied field along $X$, and the detector in the $X-Y$ plane. The measured scattering intensity, $I$, is propor- 
(a)
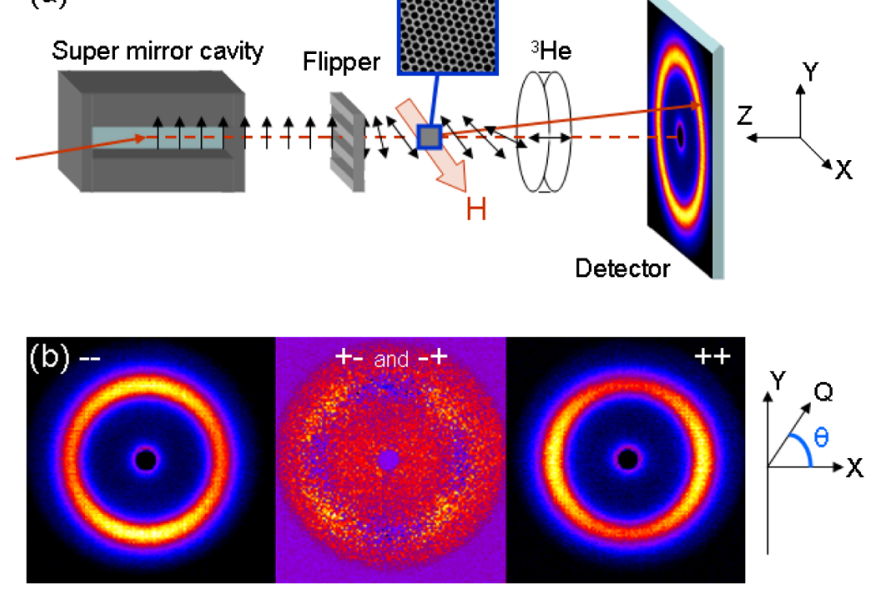

FIG. 1 (color online). (a) Experimental setup [20,21] includes a polarizing supermirror (i.e., an FeSi multilayer diffraction grating), an electromagnetic precession coil flipper, sample holder with cryostat and variable magnetic field, ${ }^{3} \mathrm{He}$ analyzer, and a position sensitive gas detector. Arrows indicate the neutron polarization direction. (b) 2D SANS images (shown here at $1.2 \mathrm{~T}, 200 \mathrm{~K})$ are corrected for polarization efficiency [21].

tional to the squared sum of the spatial nuclear and magnetic Fourier transforms defined as

$$
N, M_{J}(Q)=\sum_{K} \rho_{N, M_{J}}(K) e^{i \vec{Q} \cdot \vec{R}_{K}},
$$

where $J$ is any Cartesian coordinate, $\rho_{N, M}$ is the nuclear or magnetic scattering length density, and $\vec{R}_{K}$ is the relative position of the $K$ th scatterer. $\vec{Q}$ is the scattering wave vector. Typical, efficiency-corrected $2 \mathrm{D}$ scattering patterns from the magnetite nanoparticles in high field are shown in Fig. 1(b). From these, area-normalized sector slices of $\pm 10^{\circ}$ are taken about specific $\theta$ angles of interest in order to extract angular information, where $\theta$ is the angle between the $X$ axis and the projection of $\vec{Q}$ onto the $X-Y$ plane. The complete, angle-dependent polarization selection rules [22] simplify at several key angles and enable the unambiguous separation of $N^{2}, M_{\text {PARL }}^{2}, M_{\text {PERP }}^{2}$ as follows:

$$
\begin{gathered}
N^{2}(Q)=\frac{1}{2}\left(I_{\theta=0^{\circ}}^{++}+I_{\theta=0^{\circ}}^{--}\right), \\
M_{\mathrm{PARL}}^{2}(Q)=\frac{\left(I_{\theta=90^{\circ}}^{--}-I_{\theta=90^{\circ}}^{++}\right)^{2}}{16 N^{2}}, \\
M_{\mathrm{PERP}}^{2}(Q)=\frac{1}{6}\left(I_{\theta=0^{\circ}, 90^{\circ}}^{+-}+I_{\theta=0^{\circ}, 90^{\circ}}^{-+}\right), \\
M_{\mathrm{PARL}}^{2}(Q)=\left(I_{\theta=45^{\circ}, 135^{\circ}}^{+-}+I_{\theta=45^{\circ}, 135^{\circ}}^{-+}\right)-5 M_{\mathrm{PERP}}^{2} .
\end{gathered}
$$

Application of these equations produces the magnetic and nuclear nanoparticle scattering profiles (Fig. 2). Note that the $M_{\text {PARL }}^{2}$ profiles extracted using Eq. (3) or (5) are equivalent [Fig. 2(a)], indicating that the polarization efficiency of each element has been accurately accounted for.

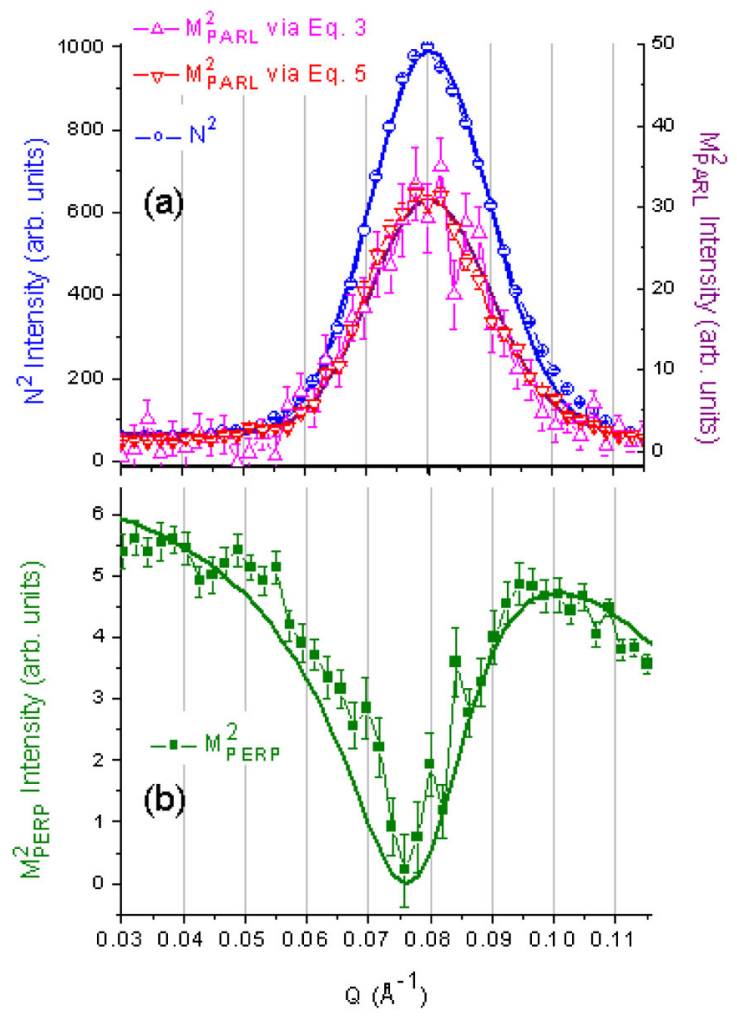

FIG. 2 (color online). Data at $1.2 \mathrm{~T}, 200 \mathrm{~K}$ scaled so that $N^{2}$ Bragg peak height is 1000 . (a) Note the intensity difference between $N^{2}$ (left axis) and $M_{\text {PARL }}^{2}$ (right axis) peaks at $0.080 \AA^{-1}$, and the equivalence of $M_{\text {PARL }}^{2}$ obtained from Eq. (5), which assumes an isotropic structure, and Eq. (3). (b) Note the prominent $M_{\text {PERP }}^{2}$ dip at $0.075 \AA^{-1}$. Solid lines show model of Fig. 3.

$N^{2}$ and $M_{\text {PARL }}^{2}$ at $1.2 \mathrm{~T}, 200 \mathrm{~K}$ have remarkably similar peak profiles centered at $0.080 \AA^{-1}$ [Fig. 2(a)], though $M_{\text {PARL }}^{2}$ is considerably smaller as expected from the known relative magnitude of $\rho_{N}=6.97 \times 10^{-6} \AA^{-2}$ and $\rho_{M}=$ $1.46 \times 10^{-6} \AA^{-2}$ (corresponding to a magnetization of $5.12 \times 10^{5} \mathrm{~A} / \mathrm{M}$ ). These scattering profiles are the product of nanoparticle packing (structure factor) with the internal nanoparticle morphology (form factor). Regarding the former, the nanoparticles are assembled into face-centered cubic (FCC) crystallites [16] of lattice length $13.6 \mathrm{~nm}$ and retain long-range FCC ordering up to the submicron level, as indicated by transmission electron microscopy imaging. Within the measured $Q$ range of $0.030 \AA^{-1}$ to $0.115 \AA^{-1}$, there are two FCC Bragg reflections, (111) and (200), which overlap and merge into a single discernable peak that becomes heavily weighted toward the (111) reflection (at $0.080 \AA^{-1}$ ) with the introduction of structural stacking faults. The convolution of the FCC structure factor [black-pink triangles Fig. 3(a)] with a $9.0 \mathrm{~nm}$ spherical form factor [black circles Fig. 3(b)], broadened to $0.020 \AA^{-1}$ (full width half maximum) to account for instrumental resolution, results in the fit (solid blue line) in Fig. 2(a). In large magnetic fields, $M_{\mathrm{PARL}}^{2}$ exhibits the same 

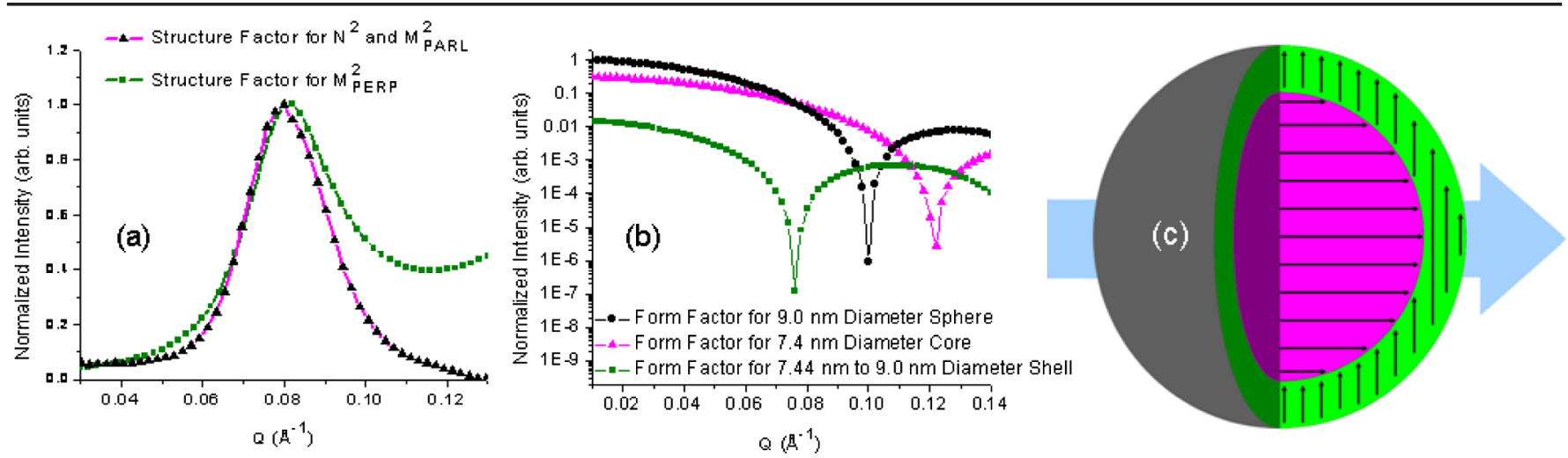

FIG. 3 (color online). (a) $N^{2}, M_{\text {PARL }}^{2}$ (black-pink curve) and $M_{\text {PERP }}^{2}$ (green curve) structure factors include instrumental resolution broadening for clarity, though in model broadening is included after convolution with respective form factors. (b) Spherical form factors produce prominent dips at specific $Q$ 's that are highly sensitive to diameter, and convoluted with curves in (a) produce the fits in Fig. 2. (c) Composite model involving a ferrimagnetic core of diameter $\approx 7.4 \mathrm{~nm}$ with a $90^{\circ}$ canted magnetic shell of thickness between $0.8 \mathrm{~nm}$ and $1.2 \mathrm{~nm}$ is depicted.

long-range structure factor of $N^{2}$ [Fig. 2(a)], but its form factor corresponds to a $7.4 \mathrm{~nm}$ sphere when $\rho_{N}$ and $\rho_{M}$ are taken into account. The product of the $M_{\text {PARL }}^{2}$ structure factor [black-pink triangles Fig. 3(a)] and form factor [pink triangles Fig. 3(b)] results in the fit (solid purple line) in Fig. 2(a).

In stark contrast with $N^{2}$ and $M_{\mathrm{PARL}}^{2}, M_{\mathrm{PERP}}^{2}$ exhibits a prominent dip at $0.075 \AA^{-1}$ [Fig. 2(b)]. It is clearly dominated by contributions from the form factor rather than the structure factor. For the structure factor, we use a hard sphere model [23] which approximates the FCC lattice structure with coherence of one to several units in extent [green squares Fig. 3(a)] in order to account for the high $Q$ scattering in Fig. 2(b). For the form factor, the $M_{\text {PERP }}^{2}$ dip [Fig. 2(b)] is classic for scattering from a spherical object [23]. However, the dip location cannot result from solid sphere scattering of diameter less than $12.0 \mathrm{~nm}$ which is unphysically large. Instead, interpreting the $M_{\text {PERP }}^{2}$ dip in terms of a shell model produces a physically reasonable average shell diameter of $8.22 \pm 0.02 \mathrm{~nm}$. A form factor shell [green squares Fig. 3(b)] of inner and outer diameters of 7.44 and $9.0 \mathrm{~nm}$, respectively, combined with a shortrange structure factor [green squares Fig. 3(a)] results in the fit (solid green line) in Fig. 2(b). To refine the shell thickness further, we take into account the relative intensity of $N^{2}$ compared with $M_{\text {PERP }}^{2}$ and apply the additional known constraints that (i) the shell resides within a $13.6 \mathrm{~nm}$ FCC lattice of nearest-neighbor spacing of $9.6 \mathrm{~nm}$, (ii) there must be at least $0.1 \mathrm{~nm}$ of surfactant beyond the nanoparticle, and (iii) $\rho_{M}$, potentially increased by uncompensated surface spins, is not allowed to deviate above bulk by more than a factor of 2 . In combination, these constraints dictate that the magnetic shell thickness must be $1.0 \mathrm{~nm} \pm 0.2 \mathrm{~nm}$.

The combined model, depicted in Fig. 3(c), is comprised of a $9.0 \mathrm{~nm}$ diameter particle with a $7.4 \mathrm{~nm}$ diameter ferrimagnetic core and a 7.44 to $9.0 \mathrm{~nm}$ perpendicular shell [Fig. 3(b)] coupled with their respective structure factors
[Fig. 3(a)]. To avoid overdetermination, we have employed the simplest model that captures the essential scattering features. While we cannot conclusively rule out a larger ferrimagnetic core of reduced $\rho_{M}$, this is far less plausible since the canted shell already contains an enhanced moment. Similarly, we cannot quantify boundary fuzziness or degree of overlap between core and shell, but we can conclude from the $M_{\text {PERP }}^{2}$ scattering that magnetic shells of nearly uniform magnitude and direction exist. Thus, models involving a disordered outermost shell [6-9], a canted moment that extends throughout the entire volume of the particle, and "hedgehoglike" [12] or "throttle" [13] symmetry do not fit $M_{\text {PERP }}^{2}$ and are excluded. The isolation of $M_{\text {PERP }}^{2}$ demonstrated here is only possible using diffraction or other spatially sensitive techniques since moments quickly average to zero across the sample, as evidenced by an equivalence of $M_{Y}^{2}$ and $M_{Z}^{2}$ [22].

The magnetic shell evolution was investigated for a series of temperatures at 1.2 Tesla [Fig. 4(a)]. Zero-field cooling to $10 \mathrm{~K}$ before application of the applied field causes all $M_{\mathrm{PERP}}^{2}$ scattering from the ordered magnetic shell to vanish (though this does not preclude the possibility of a disordered shell). Increasing the temperature to $160 \mathrm{~K}$ produces a $M_{\text {PERP }}^{2}$ dip position of $\approx 0.075 \AA^{-1}$, which is essentially the same as at $200 \mathrm{~K}$. Increasing the temperature further to 300 or $320 \mathrm{~K}$ reproducibly shifts this dip to a higher $Q$ of $\approx 0.082 \AA^{-1}$. Assuming that the shell extends to the outer edge of the nanoparticle, as is the case at $200 \mathrm{~K}$, the $M_{\text {PERP }}^{2}$ dips at $160 \mathrm{~K}$ and $300-320 \mathrm{~K}$ correspond to shells of thickness 1.0 and $1.5 \mathrm{~nm}$ [solid curves in Fig. 4(a)], respectively.

In a remanent field of $0.005 \mathrm{~T}$ at $300 \mathrm{~K}$ virtually no nearest-neighbor interparticle correlations persist [21], which makes this an ideal condition to probe magnetic morphology with negligible influence from nearestneighbor nanoparticles. Here the $M_{\mathrm{PERP}}^{2}=M_{\mathrm{PARL}}^{2}$ scattering [Fig. 4(b)] is consistent with a ferrimagnetic sphere of diameter $9.0 \mathrm{~nm} \pm 0.2 \mathrm{~nm}$, indicating that neither a canted 


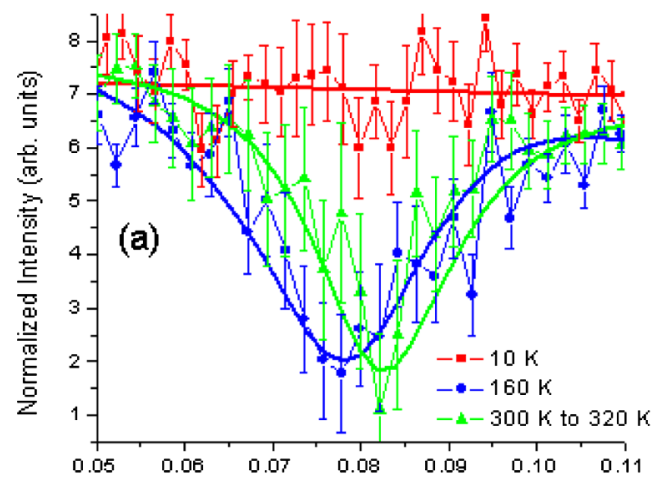

$Q\left(\mathrm{~A}^{-1}\right)$

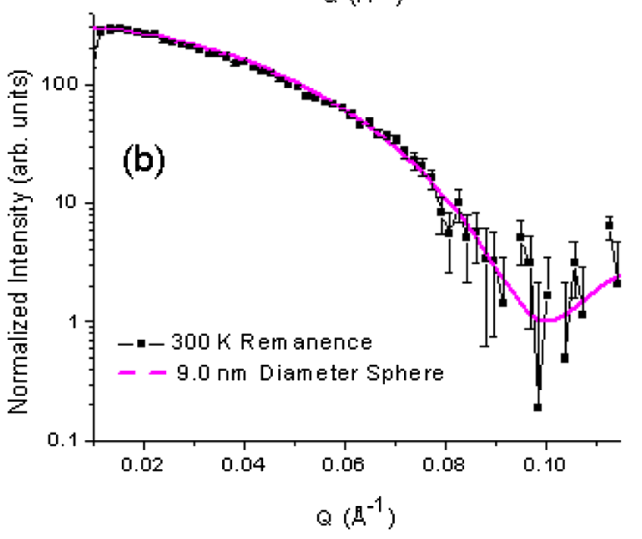

FIG. 4 (color online). Field and temperature dependence of $M_{\text {PERP. (a) Varying the temperature in an applied field of } 1.2 \mathrm{~T}}^{2}$ indicates the presence of canted shells at 160, 300, and $320 \mathrm{~K}$ (the latter two similar enough to be averaged), but no ordered shell when zero-field cooled to $10 \mathrm{~K}$ before application of the field. (b) At $300 \mathrm{~K}$ in a remanent field of $0.005 \mathrm{~T}$ the magnetic scattering is well modeled by a uniform sphere of diameter $9.0 \mathrm{~nm}$ lacking shell features.

nor disordered magnetic shell of thickness more than $0.1 \mathrm{~nm}$ exists. (We may not be able to distinguish between a fully ferrimagnetic structure and a nearly uniform one with coherent edge effects, such as an artichoke structure [13].) These remanent and temperature dependent data clearly demonstrate that the formation of a canted shell is magnetic, rather than chemical, in origin.

In conclusion, we have expanded the capability of polarization analyzed SANS to directly probe the 3D magnetic morphology of nanoparticles in any field. Applied to $9.0 \mathrm{~nm}$ magnetite nanoparticles, this resulted in the discovery of magnetic shells 1.0 to $1.5 \mathrm{~nm}$ thick, canted $90^{\circ}$ to their ferrimagnetic cores under conditions of nominal saturation at temperatures of $160 \mathrm{~K}$ or more. The concept of a chemically uniform, but magnetically distinct, core and canted shell is not without precedent $[14,24]$, though not previously reported experimentally. We speculate that the lack of radial symmetry arises primarily from the competition between interparticle coupling within the nanoparticle array [21] (that sets a net direction) and nanoparticle surface anisotropy $[12,13]$ (that promotes outward spin canting). For example, experiment [3] has shown that intraparticle exchange biasing can be altered by varying nanoparticle packing distance. Thus, dipolar interparticle coupling may play a significant role in determining the magnetic core-shell morphology and could explain the selection of a locally preferred orientation of the magnetic shells within small regions. This unique observation of ordered, canted magnetic shells within chemically uniform particles underscores the complex magnetic interactions present within these nanoparticle systems.

This work utilized facilities supported in part by National Science Foundation grants DMR-0454672, DMR-0704178, and DMR-0804779 and Department of Energy grant DE-FG02-08ER40481. Development of the ${ }^{3} \mathrm{He}$ spin filters was supported in part by the Department of Energy. We would like to thank Cedric Gagnon of the NIST Center for Neutron Research for his efforts in making this experiment successful.

[1] R. A. Kodama and A. E. Berkowitz, Phys. Rev. B 59, 6321 (1999).

[2] Y. Ijiri et al., Appl. Phys. Lett. 86, 243102 (2005).

[3] J. Noguès et al., Phys. Rev. Lett. 97, 157203 (2006).

[4] S. E. Inderhees et al., Phys. Rev. Lett. 101, 117202 (2008).

[5] I. V. Golosovsky et al., Phys. Rev. Lett. 102, 247201 (2009).

[6] P. Dutta et al., J. Appl. Phys. 105, 07 B501 (2009).

[7] J. Curiale et al., Appl. Phys. Lett. 95, 043106 (2009).

[8] A. Kovacs et al., Phys. Rev. Lett. 103, 115703 (2009).

[9] C. Westman et al., J. Phys. D 41, 225003 (2008).

[10] R. Yanes et al., Phys. Rev. B 76, 064416 (2007).

[11] J. Mazo-Zuluaga, J. Restrepo, and J. Majía-López, Physica (Amsterdam) 398B, 187 (2007).

[12] J. Mazo-Zuluaga et al., J. Appl. Phys. 105, 123907 (2009).

[13] L. Berger et al., Phys. Rev. B 77, 104431 (2008).

[14] Y. Hu A and Du, J. Nanosci. Nanotechnol. 9, 5829 (2009).

[15] S. Sun et al., J. Am. Chem. Soc. 126, 273 (2004).

[16] D. A. Farrell et al., J. Magn. Magn. Mater. 303, 318 (2006).

[17] T. R. Gentile et al., J. Appl. Crystallogr. 33, 771 (2000).

[18] A. Wiedenmann, Physica (Amsterdam) 356B, 246 (2005).

[19] A. M. Gaspar et al., Biochim. Biophys. Acta 1804, 76 (2010).

[20] W.C. Chen et al., Physica (Amsterdam) 404B, 2663 (2009).

[21] K. L. Krycka et al., Physica (Amsterdam) 404B, 2561 (2009).

[22] See supplementary material at http://link.aps.org/ supplemental/10.1103/PhysRevLett.104.207203.

[23] A. Guinier, X-Ray Diffraction in Crystals, Imperfect Crystals, and Amorphous Bodies (Dover, New York, 1994).

[24] N. N. Phuoc et al., Phys. Status Solidi B 244, 4518 (2007). 